\title{
Drug-Resistant Hyponatremia after Escitalopram Intake: A Series of Two Case Reports
}

\author{
Justyna Kacperczyk ${ }^{1}$, Adrian Perdyan², Małgorzata Stompór ${ }^{3,4}$ \\ ${ }^{1}$ Students' Scientific Society of Geriatrics, University of Warmia and Mazury, Olsztyn, Poland \\ ${ }^{2}$ Faculty of Medicine, Medical University of Gdansk, Gdansk, Poland \\ ${ }^{3}$ Geriatric Outpatient Clinic, Dobre Miasto, Poland \\ ${ }^{4}$ Faculty of Medicine, University of Warmia and Mazury, Olsztyn, Poland
}

Corresponding Author:

Adrian Perdyan, MD

Faculty of Medicine, Medical University of Gdansk, Marii Skłodowskiej-Curie Street 3a, Gdansk 80-210, Poland

E-mail: perdyan.adrian@gumed.edu.pl ORCID:

https://orcid.org/0000-0002-5098-4357

Received: June 14, 2021

Revised: August 25, 2021

Accepted: September 17, 2021
The consumption of new selective serotonin reuptake inhibitors (SSRIs) is raising dramatically especially in European countries. It contributes to occurrence of clinically important drug side effects. One of which can be hyponatremia. We present two case reports of 85-year-old and 84-year-old women who developed hyponatremia after escitalopram administration. We hypothesize that in both cases hyponatremia was connected with antidepressants administration. However, due to multiple comorbidities and polypharmacy it is often impossible to establish the exact mechanism of hyponatremia. Moreover, it is crucial to distinguish subtypes of drug-induced syndrome of inappropriate antidiuretic hormone hypersecretion (SIADH), such as SSRI-induced SI$A D H$, reset osmostat SIADH, thiazide-associated hyponatremia, thiazide-induced hyponatremia, mineralocorticoid responsive hyponatremia of older adults, in order to properly diagnose and treat geriatric patients. Administration of antidepressants or thiazides should be followed by a regular monitoring of serum sodium level.

Key Words: Hyponatremia, Serotonin uptake inhibitors, Geriatrics, Case reports

\section{INTRODUCTION}

Mental health problems have been increasing worldwide in recent years. Thus, an inevitable increase in the consumption of antidepressants is expected soon, which is predicted to be much greater than that observed at the beginning of the current century, ${ }^{1,2)} \mathrm{Si}$ multaneously, increased rates of clinically important side effects of new and old psychiatric drugs will also occur. Drug-induced hyponatremia was previously reported after the administration of fluoxetine, sertraline, paroxetine, and fluvoxamine ${ }^{3)}$ and has also recently been described for escitalopram, a new selective serotonin reuptake inhibitor (SSRI).

The prevalence of hyponatremia among hospitalized older adult (age $>75$ years) patients ranges from $11.6 \%$ to $42.6 \%$ and up to $22.5 \%$ among patients in long-term care facilities. ${ }^{6}$ Hyponatremia leads to a lack of stability, frequent uncontrolled falls, bone frac- tures, and deterioration of cognitive functioning. ${ }^{1)}$ Although a serum sodium level (SSL) of $135 \mathrm{mmol} / \mathrm{L}$ is considered within the normal range, increased hospital mortality is observed for levels below $138 \mathrm{mmol} / \mathrm{L} .^{5)}$ Moreover, managing hyponatremia can be challenging due to a variety of potential causes (Table 1 ). Thus, this condition should be distinguished from other ailments such as motoric cognitive risk syndrome, which is common among older adults and may also manifest in falls. ${ }^{7)}$ Our report highlights the possible life-threatening side effects of seemingly safe medications that are widely used in clinics.

\section{CASE REPORT}

\section{Case Study 1}

An 85-year-old woman with symptoms of cognitive impairment (CI) was admitted to the geriatric outpatient clinic in October 
Table 1. Etiology of hyponatremia

\begin{tabular}{|c|c|}
\hline & Symptom \\
\hline \multirow[t]{2}{*}{ Hypovolemic } & Extrarenal loss (For example, diarrhea, vomiting, and burns) \\
\hline & $\begin{array}{l}\text { Renal loss (For example, diuretics use, nephropathy, osmotic diuresis osmotic diuresis induced by excessive excretion of glucose or urea or } \\
\text { urea induced) }\end{array}$ \\
\hline \multirow[t]{6}{*}{ Euvolemic } & SIADH (For example, due to cancer, use of drugs, CNS-related disorders, pulmonary diseases, stress, hereditary factors) \\
\hline & Thiazides-induced \\
\hline & Hypothyroidism \\
\hline & Adrenal insufficiency \\
\hline & Psychiatric diseases (polydipsia) \\
\hline & Alcohol abuse \\
\hline \multirow[t]{4}{*}{ Hypervolemic } & Heart failure \\
\hline & Liver cirrhosis \\
\hline & Nephrotic syndrome \\
\hline & Renal failure \\
\hline
\end{tabular}

SIADH, syndrome of inappropriate secretion of antidiuretic hormone; CNS, central nervous system.

2015. She had multiple comorbidities, including arterial hypertension $(\mathrm{AH})$, cholecystolithiasis, and osteoarthritis, and was prescribed amlodipine, omeprazole, hydroxyzine, piracetam, and vitamin D3 (the treatment characteristics and disease timeline are detailed in Table 2). Throughout the diagnostic process, head computed tomography (CT) images showed cortical and subcortical atrophy. The patient's thyroid function, serum electrolyte, and vitamin B12 levels were all within the normal ranges. Comprehensive Geriatric Assessment (CGA) resulted in the diagnosis of mild dementia with no functional disability and low risk of falls. Due to the diagnosis of Alzheimer disease, memantine was also prescribed. In April 2017, the patient was admitted with behavioral and psychological symptoms of dementia (BPSD). Escitalopram ( $5 \mathrm{mg} /$ day) was administered. Meanwhile, general practitioners modified the treatment for hypertension and added indapamide and ramipril. During the next 12 months, a major improvement was observed with no signs of BPSD. In April 2018, the patient experienced a lack of stability and fell. Blood tests revealed hyponatremia (serum sodium level [SSL], $129 \mathrm{mmol} / \mathrm{L}$ ), with no signs of hyper- or hypovolemia. Escitalopram was withdrawn due to suspicion of the potential side effects of SSRI use. The patient was prescribed oral sodium chloride and fluid restriction was recommended. In addition, quetiapine and doxepin were added to manage the patient's symptoms of agitation, anxiety, and somatization. Despite the best possible care in the following months, the SSL was labile, which prompted the withdrawal of indapamide, until the next fall occurred in August 2019, when she was diagnosed with non-ST elevation myocardial infarction and an SSL of $123 \mathrm{mmol} / \mathrm{L}$. The patient's follow-up lasted until July 2020 without new complaints; however, once induced, the hyponatremia remained constant de- spite the sodium levels being in the normal range for short periods.

\section{Case Study 2}

An 84-year-old woman was admitted to the geriatric outpatient clinic in April 2016 with symptoms of CI, restlessness, and anxiety. Her medical history included gastroesophageal reflux disease and essential head tremor. Head CT images showed cortical-subcortical atrophy and multiple focal hypodense lesions. The patient's pharmacological treatment before admission consisted of atorvastatin, lutein, pantoprazole, and ginkgo biloba. Her thyroid function, serum electrolytes, and vitamin $B_{12}$ levels were within the normal ranges. In CGA, the patient was diagnosed with $\mathrm{CI}$ and vascular dementia (the treatment characteristics and disease timeline are detailed in Table 3). In April 2017, the patient was admitted with symptoms of depressive episodes and anxiety. She was prescribed alprazolam with escitalopram $(5 \mathrm{mg} /$ day $)$. Three months later, the patient was admitted after a fall with an SSL of $131 \mathrm{mmol} / \mathrm{L}$ despite being euvolemic. The previously administered pharmacotherapy was sustained, sodium chloride (orally) was prescribed, and fluid restriction was recommended. In 10 months of follow-up, the patient was admitted to the neurology department with transient speech disorder after another fall. Head CT images showed no new findings; however, laboratory tests showed hyponatremia $(124 \mathrm{mmol} / \mathrm{L})$ despite the discontinuation of escitalopram. In the next 3 months, the SSL remained below the normal range. The supplementation of sodium chloride was changed to fludrocortisone acetate because of nausea. During this treatment, the SSL normalized, but her blood pressure (BP) increased to $170 / 100 \mathrm{mmHg}$ and lercanidipine was prescribed. In January 2019, the patient's SSL was $135 \mathrm{mmol} / \mathrm{L}$ and her BP was 
Table 2. Course of treatment of Patient 1

\begin{tabular}{|c|c|c|c|}
\hline Date of visit & Pharmacotherapy & Frequency of drug-induced hyponatremia (\%) & Serum sodium level (mmol/L) \\
\hline \multirow[t]{4}{*}{ Oct 2015} & 2,000 UI vitamin D3 qd & 0 & 139 \\
\hline & $2.5 \mathrm{mg}$ amlodipine $\mathrm{qd}$ & 0 & \\
\hline & 10 mg hydroxyzine qd & 0 & \\
\hline & $1,200 \mathrm{mg}$ of piracetam bid (withdrawn during the first visit) & 0 & \\
\hline Nov 2015 & +5 mg memantine qd & 0 & - \\
\hline \multirow[t]{5}{*}{ Apr 2017} & New treatment regimen: & & - \\
\hline & $2.5 \mathrm{mg}$ ramipril qd & Unknown frequency & \\
\hline & $5 \mathrm{mg}$ amlodipine $\mathrm{qd}$ & 0 & \\
\hline & $1.5 \mathrm{mg}$ indapamide qd & $1-5$ & \\
\hline & $10 \mathrm{mg}$ memantine qd & Unknown frequency & \\
\hline \multirow[t]{2}{*}{ Sep 2018} & $+25 \mathrm{mg}$ quetiapine qd & $0.1-1$ & 124 \\
\hline & +10 mg doxepin qd & 0 & \\
\hline Apr 2019 & -1.5 mg indapamide & - & 129 \\
\hline Aug 2019 & $+2.5 \mathrm{mg}$ torasemide $\mathrm{qd}$ & 0 & 123 \\
\hline Jul 2020 & The end of follow-up & - & 129 \\
\hline
\end{tabular}

qd, quaque die (daily); bid, twice daily; +, drug added; -, drug withdrawn.

Table 3. Course of treatment of Patient 2

\begin{tabular}{|c|c|c|c|}
\hline Date of visit & Pharmacotherapy & Frequency of drug-induced hyponatremia (\%) & Serum sodium level (mmol/L) \\
\hline \multirow[t]{5}{*}{ Apr 2016} & $40 \mathrm{mg}$ atorvastatin $\mathrm{qd}$ & 0 & 139 \\
\hline & 20 mg pantoprazole qd & Unknown frequency & \\
\hline & lutein & 0 & \\
\hline & ginkgo biloba & 0 & \\
\hline & +75 mg acetylsalicylic acid qd & 0 & \\
\hline \multirow[t]{2}{*}{ Aug 2017} & $+0.25 \mathrm{mg}$ alprazolam ad hoc & 0 & - \\
\hline & +5 mg escitalopram qd & Unknown frequency & \\
\hline Nov 2017 & $+2 \mathrm{~g}$ natrium chloride bid & 0 & 131 \\
\hline \multirow[t]{2}{*}{ Sep 2018} & -escitalopram, natrium chloride & - & 124 \\
\hline & +10 mg hydroxyzine ad hoc & 0 & \\
\hline Oct 2018 & $+0.1 \mathrm{mg}$ fludrocortisone acetate $\mathrm{qd}$ & 0 & 132 \\
\hline Nov 2018 & +10 mg lercanidipine qd & 0 & 139 \\
\hline Jul 2020 & The end of follow-up & - & 135 \\
\hline
\end{tabular}

qd, quaque die (daily); bid, twice daily; +, drug added; -, drug withdrawn.

normal. Until July 2020, her SSL was within the normal range.

We obtained informed consent from the participants before their participation.

\section{DISCUSSION}

We present two case reports of women aged 85 and 84 years, re- spectively, who developed hyponatremia, most likely after escitalopram administration (escitalopram-induced syndrome of inappropriate secretion of antidiuretic hormone $[\mathrm{SIADH}])$. The lack of symptoms of hypervolemia at the time of initial diagnosis excluded kidney, thyroid, cardiovascular, and adrenal cortex disorders, as well as neoplasms as other possible explanations.

A few types of SIADH have been described in the literature. 
First, syndromes associated with excessive $\mathrm{ADH}$ production are usually caused by antidepressants (SSRIs, SNRIs, serotonin and noradrenaline reuptake inhibitors, and tricyclic antidepressants [TCAs]), antipsychotics, antiepileptics, and antineoplastic drugs. ${ }^{1,6,8)}$ Furthermore, many drugs, including carbamazepine, cyclophosphamide, and nonsteroidal anti-inflammatory drugs, can induce hyponatremia by the peripheral receptor effect rather than by stimulating the secretion of the hormone itself. ${ }^{8)}$ Moreover, reset osmostat (RO) SIADH in older adults might also occur due to alterations in osmoreceptor cell metabolism. ${ }^{9)}$ The condition is frequently observed in residents of long-term care facilities with simultaneous quadriplegia, psychosis, cerebral hemorrhage, dementia with Lewy bodies, alcoholism, malnutrition, malignancy, and infections." Lastly, hyponatremia after the administration of thiazide or thiazide-like diuretics can occur in up to $30 \%$ of older adult patients. ${ }^{10)}$ Advanced age, female sex, and low body mass index have been identified as risk factors for the development of thiazide-associated hyponatremia.

Establishing an exact diagnosis for the first patient was challenging because of the use of multiple drugs that could cause SIADH (memantine, quetiapine, and doxepin). ${ }^{8,11)}$ In the second patient, hyponatremia seemed to be more directly connected with the use of escitalopram owing to the lack of other diseases or drugs that could contribute to the development of SIADH. SSRI-induced SI$\mathrm{ADH}$ is one of the most common mechanisms of hyponatremia in older adults, although only 14 cases of escitalopram-induced SI$\mathrm{ADH}$ have been described in the literature. ${ }^{1,11,12)}$ However, it must be emphasized that in the presented cases, even small doses of escitalopram $(5 \mathrm{mg})$ caused serious side effects. In other reports, the doses of escitalopram were usually $10 \mathrm{mg}{ }^{11)}$ Recent reports suggest a lower risk of hyponatremia with the use of TCAs and noradrenergic and specific serotonergic antidepressants compared to SSRI and SNRI; thus, changing SSRI to TCA (doxepin) might have been reasonable. ${ }^{12,13)}$

In both patients, the hyponatremia did not resolve after escitalopram withdrawal or standard treatment. In the second patient, SSL normalization was achieved after treatment with fludrocortisone acetate. According to the available data, the SSL should normalize approximately 2 weeks after SSRI discontinuation and the introduction of limited fluid intake and sodium chloride supplementation (as stated by guidelines). ${ }^{6,13)}$ However, despite this treatment, hyponatremia persisted in our patients for months. Abnormal homeostatic mechanisms, such as RO syndrome, may explain the observed resistance to treatment. This theory is supported by the fact that hyponatremia due to $\mathrm{RO}$ does not respond to fluid restriction (as non-RO SIADH hyponatremia), oral sodium supplementation, or fludrocortisone treatment. ${ }^{9)}$ Another explanation could be adrenal insufficiency or mineralocorticoid-responsive hyponatremia of the elderly (MRHE), which requires the exclusion of SI$\mathrm{ADH}$ and adrenal dysfunction. ${ }^{14)} \mathrm{MRHE}$ is considered a mildly hypovolemic hyponatremia caused by renal sodium loss.

Age-related decreased sodium reabsorption by proximal renal tubules and hyporesponsiveness of the renin-angiotensin-aldosterone system may cause constantly increased urinary sodium excretion. Decreased sodium retention leads to volume depletion, which causes elevation of plasma antidiuretic hormone levels. The recommended treatment for MRHE is, instead of water restriction, which may worsen hyponatremia, an administration of mineralocorticoids such as fludrocortisone acetate. Unfortunately, both fludrocortisone treatment and dietary sodium chloride supplementation may be limited by an increase in BP.

In conclusion, managing hyponatremia in older adults is complex. Regular monitoring of SSLs should be mandatory in this population, particularly following the initiation of antidepressants or thiazides. Unfortunately, due to the unpredictability of drug-related hyponatremia, the timing or frequency of such monitoring cannot be precisely defined and should be based on clinical experience. In addition to informing patients and their caregivers of the potential risks of hyponatremia, preventive fluid moderation or restriction and ordering SSL assessment should be considered. Treatment of hyponatremia requires a multimodal approach that considers patient comorbidities and pharmacotherapy. However, despite the best care, it can last for months. Reporting side effects, especially of the most popular drugs to the local center for drug monitoring, is crucial in terms of increasing our awareness of the benefits of our patients.

\section{ACKNOWLEDGMENTS}

\section{CONFLICT OF INTEREST}

The researchers claim no conflicts of interest.

\section{FUNDING}

None.

\section{AUTHOR CONTRIBUTION}

Conceptualization, AP, JK, MS; Data curation, AP, JK, MS; Funding acquisition, AP, JK, MS; Investigation, AP, JK; Methodology, JK, MS; Project administration, JK, MS; Supervision, MS; Writing-original draft, JK, AP, MS; Writing-review \& editing, JK, AP.

\section{REFERENCES}

1. Leth-Moller KB, Hansen AH, Torstensson M, Andersen SE, 
Odum L, Gislasson G, et al. Antidepressants and the risk of hyponatremia: a Danish register-based population study. BMJ Open 2016;6:e11200.

2. Hossain MM, Tasnim S, Sultana A, Faizah F, Mazumder H, Zou L, et al. Epidemiology of mental health problems in COVID-19: a review. F1000Res 2020;9:636.

3. Grover S, Biswas P, Bhateja G, Kulhara P. Escitalopram-associated hyponatremia. Psychiatry Clin Neurosci 2007;61:132-3.

4. Gankam-Kengne F, Ayers C, Khera A, de Lemos J, Maalouf NM. Mild hyponatremia is associated with an increased risk of death in an ambulatory setting. Kidney Int 2013;83:700-6.

5. Wald R, Jaber BL, Price LL, Upadhyay A, Madias NE. Impact of hospital-associated hyponatremia on selected outcomes. Arch Intern Med 2010;170:294-302.

6. Burst V. Etiology and epidemiology of hyponatremia. Front Horm Res 2019;52:24-35.

7. Meiner Z, Ayers E, Verghese J. Motoric cognitive risk syndrome: a risk factor for cognitive impairment and dementia in different populations. Ann Geriatr Med Res 2020;24:3-14.

8. Liamis G, Milionis H, Elisaf M. A review of drug-induced hypo- natremia. Am J Kidney Dis 2008;52:144-53.

9. Feder J, Gomez JM, Serra-Aguirre F, Musso CG. Reset Osmostat: facts and controversies. Indian J Nephrol 2019;29:232-4.

10. Burst V, Grundmann F, Kubacki T, Greenberg A, Becker I, Rudolf $\mathrm{D}$, et al. Thiazide-associated hyponatremia, report of the hyponatremia registry: an observational multicenter international study. Am J Nephrol 2017;45:420-30.

11. Raj R, Jacob A, Venkatanarayan A, Doraiswamy M, Ashok M. Severe symptomatic hyponatremia secondary to escitalopram-induced SIADH: a case report with literature review. Case Rep Nephrol 2018;2018:3697120.

12. Lien YH. Antidepressants and hyponatremia. Am J Med 2018; 131:7-8.

13. Gandhi S, Shariff SZ, Al-Jaishi A, Reiss JP, Mamdani MM, Hackam DG, et al. Second-generation antidepressants and hyponatremia risk: a population-based cohort study of older adults. Am J Kidney Dis 2017;69:87-96.

14. Katayama K, Tokuda Y. Mineralocorticoid responsive hyponatremia of the elderly: a systematic review. Medicine (Baltimore) 2017;96:e7154. 